\title{
An Extended Robust H infinity Filter for Nonlinear Uncertain Systems with Constraints
}

\author{
Jaewon Seo, Member, IEEE, Myeong-Jong Yu, Chan Gook Park, Member, IEEE, and Jang Gyu Lee, \\ Member, IEEE
}

\begin{abstract}
In this paper, a robust filter is proposed to effectively estimate the system states in the case where system model uncertainties as well as disturbances are present. The proposed robust filter is constructed based on the linear approximation methods for a general nonlinear uncertain system with an integral quadratic constraint. We also derive the important characteristic of the proposed filter, a modified $\mathrm{H}_{\infty}$ performance index. Analysis results show that the proposed filter has robustness against disturbances such as process and measurement noises, and against parameter uncertainties. Simulation results show that the proposed filter effectively improves the performance.
\end{abstract}

Index Terms - extended robust $\mathbf{H}_{\infty}$ filter, nonlinear estimation, nonlinear uncertain system, robust estimation

\section{INTRODUCTION}

$\mathrm{D}$ uring the last four decades, the Kalman filter and the extended Kalman filter (EKF) have been widely used in the estimation problems. They require not only a precise system model, but also the statistical property of the noise to achieve accurate performance. However, model uncertainty and incomplete statistical information are often encountered in real applications and make it difficult to precisely estimate the system states, potentially leading to very large estimation errors. These difficulties can be overcome by studying a robust filter $[1,2,3]$.

Recently, a robust filter has received considerable attention. It has robustness against 1) unknown statistics of the noise processes, and 2) system model uncertainty. They can be categorized as $\mathrm{H}_{2}$ filters, $\mathrm{H}_{\infty}$ filters, and mixed $\mathrm{H}_{2} / \mathrm{H}_{\infty}$ filters [4,5,6,7]. The $\mathrm{H}_{\infty}$ filter minimizes the $\mathrm{H}_{\infty}$ norm of the transfer function between the noise and the estimation error. Thus, the $\mathrm{H}_{\infty}$ filter is usually employed when the energy of the system noise is bounded and the statistical

Manuscript received Aug. 26, 2005. This work was supported in part by Automatic Control Research Center in Seoul National University, Electronics Telecommunications Research Institute, and BK-21 program in Korea.

Jaewon Seo and Jang Gyu Lee are with the School of Electrical Engineering and Computer Science, Seoul National University, Seoul, Korea (e-mail: jwseo1@snu.ac.kr; jgl@snu.ac.kr).

Myeong-Jong Yu is with the Agency for Defense Development, Daejeon, Korea

Chan Gook Park is with the School of Mechanical and Aerospace Engineering, Seoul National University, Seoul, Korea (corresponding author: phone: 822-880-1675; fax: 822-888-7958; e-mail: chanpark@snu.ac.kr). properties of the noise are unknown. This filter minimizes the highest energy gain of the estimation error for all initial conditions and noises. In particular, a robust $\mathrm{H}_{\infty}$ filter, a robust filter with a modified $\mathrm{H}_{\infty}$ performance, can be established for a system with model uncertainty as well as unknown statistical noise properties [3].

For a nonlinear system, an extended Kalman filter has been widely used. For the design of the EKF, with the help of the Taylor expansion, a nonlinear system is linearized and approximated. Therefore, when it is applied to a highly nonlinear system, excessive estimation errors can occur. The EKF requires statistical information about the noise inputs, e.g. whether or not they are white, what kinds of probability density functions (PDFs) they have. In many cases, it is not easy to obtain the information. Several studies on the nonlinear robust filter have been accomplished $[3,8,9,10,15]$. The $\mathrm{H}_{\infty}$ nonlinear filter with Hamilton- Jacobi inequality (HJI) is one result of such studies, but its computation procedures for obtaining the filter are somewhat complicated [8]. To avoid complicated computation procedures, an approximated solution to the robust filtering problem has been recently developed based on a linearization method. The robust filter derived by this approach is called the extended robust filter or extended $\mathrm{H}_{\infty}$ filter [3,9]. In [10], the nonlinear state estimation by an approximation is proposed for a nonlinear system with uncertainties described by an integral quadratic constraint (IQC). In that, a set-valued state estimation is given firstly, and then a Kalman filter-like characterization of the set-valued state estimation is presented. That characterization makes the estimator simple and familiar because its structure resembles that of the EKF.

In this paper, a robust $\mathrm{H}_{\infty}$ filter for nonlinear uncertain systems with an integral quadratic constraint is derived based on the approach of [10]. For the filter derivation, a set-valued state estimate is introduced and it is approximated on the local solution. Then $\mathrm{H}_{\infty}$ filter-like structure is established. The system has additive disturbance inputs, and the disturbances must satisfy the IQC. Then the performance index of the filter is proposed and analyzed. Similarly, Zhang, et al., also proposed a robust $\mathrm{H}_{\infty}$ filter for nonlinear uncertain systems in [8]. The robust filter is obtained by solving a second-order nonlinear HJI. It has no constraint such as the IQC for the disturbance inputs, but has multiplicative white noise. For a class of special nonlinear systems, a linear matrix inequality-based algorithm is used for the robust filter design. It is much easier than the algorithm for HJI.

The paper consists of the following Sections. In Section 2, the problem to be solved is presented, and Section 3 derived the extended robust $\mathrm{H}_{\infty}$ filter. The performance index for the proposed filter is derived in Section 4, and the example is given in Section 5. 
The conclusion is in Section 6.

\section{PROBLEM FORMULATION}

Consider a nonlinear uncertain system described by

$$
\dot{x}(t)=f(x(t))+B_{1}(t) \Delta_{1}(t) N(x(t))+B_{2}(t) w_{0}(t)
$$

$$
y(t)=h(x(t))+\Delta_{2}(t) N(x(t))+v_{0}(t) .
$$

In the above, $x(t) \in R^{n}$ is the state, $y(t) \in R^{m}$ is the measurement, and $f(x(t))$ and $h(x(t))$ are nonlinear system dynamic and observation model, respectively, which are of class $C^{1}$ with bounded first derivative. $w_{0}(t)$ and $v_{0}(t)$ are the process and measurement noises, and their statistical properties are unknown. $B_{1}(t) \Delta_{1}(t) N(x(t))$ and $\Delta_{2}(t) N(x(t))$ represent the system model uncertainties. $B_{1}(t)$ and $N(x(t))$ are known matrices. $\Delta_{1}(t)$ and $\Delta_{2}(t)$ are unknown matrices satisfying the condition

$$
\left\|\begin{array}{l}
Q_{1}^{-1 / 2} \Delta_{1}(t) \\
R_{1}^{-1 / 2} \Delta_{2}(t)
\end{array}\right\| \leq 1
$$

where $Q_{1}$ and $R_{1}$ are bounded positive definite matrices.

By converting the uncertainties to the fictitious noises and introducing a freedom parameter, the uncertain system (1) and (2) can be transformed into,

$$
\begin{aligned}
& \dot{x}(t)=f(x(t))+B(t) w(t) \\
& y(t)=h(x(t))+v(t)
\end{aligned}
$$

where $B(t)=\left[\begin{array}{ll}\varepsilon B_{1}(t) & B_{2}(t)\end{array}\right], \quad n(t)=\varepsilon^{-1} N(x(t)) \quad$ ， $w(t)=\left[\begin{array}{c}\Delta_{1}(t) n(x(t)) \\ w_{0}(t)\end{array}\right], v(t)=\left[\begin{array}{ll}I & I\end{array}\right]\left[\begin{array}{c}\varepsilon \Delta_{2}(t) n(x(t)) \\ v_{0}(t)\end{array}\right]$, and $\varepsilon$

is a free parameter. For the nonlinear uncertain system (3) and (4), we will design a robust filter which has an $\mathrm{H}_{\infty}$ filter-like structure. The filter output is of the form

$$
z(x(t))=\left[\begin{array}{ll}
(L(t) x(t))^{T} & (\gamma n(t))^{T}
\end{array}\right]^{T}
$$

where $\gamma$ is a given positive real value that indirectly indicates the level of noise attenuation in this robust filter design.

For the nonlinear uncertain system, suppose that the following constraint is satisfied $[10,11]$.

$$
\Phi(x(0))+\int_{0}^{T} L_{1}(w, v) d t \leq d+\int_{0}^{T} L_{2}(n, z) d t
$$

where $0 \leq t \leq T$ and $d$ is an assigned positive real number. For the system (3)-(5) and the constraint (6), the following assumptions are required. [10]

Assumption 1: Every function shown in (3)-(6) belongs to $C^{1}$ and the first derivative is bounded.

Assumption 2: The matrix $N(x(t))$ is bounded.

Assumption 3: The functions $\Phi, L_{1}$, and $L_{2}$ are bounded nonnegative functions. They also satisfy

$$
\left|\phi\left(x_{2}\right)-\phi\left(x_{1}\right)\right| \leq \theta\left(1+\left|x_{2}\right|+\left|x_{1}\right|\right)\left|x_{2}-x_{1}\right|
$$

where $\theta>0$ and $\phi=\Phi, L_{1}$, or $L_{2}$.

Assumption 4: The function $L_{1}$ satisfies a coercivity condition,

$$
L_{1}(w, v) \geq c|w|^{2} \text { where } c>0 \text {. }
$$

\section{EXTENDED ROBUST $\mathrm{H}_{\infty}$ FILTER}

In this section, an extended robust $\mathrm{H}_{\infty}$ filter based on a local solution of the filtering problem is derived by linearizing the system in the neighborhood of the estimated trajectory, $\hat{x}$. This approach for the extended robust $\mathrm{H}_{\infty}$ filter is similar to that of [10]. Through this approach, an $\mathrm{H}_{\infty}$ filter-like characterization of a set-valued state estimation is obtained.

Theorem 1[10] : Assume that the uncertain system (3), (4) with (6) satisfies Assumptions 1 4. Then, the corresponding set of possible states is given by

$$
\chi_{s}=\left\{x \in R^{n}: V(x, s) \leq d\right\}
$$

where $V(x, t)$ is the unique viscosity solution of (8) in $C\left(R^{n} \times[0, s]\right)$.

$$
\begin{aligned}
& \frac{\partial}{\partial t} V+\max _{w}\left[\nabla_{x} V(f(x)+B w)\right. \\
& \left.-L_{1}(w, v)+L_{2}(n(x), z(x))\right]=0
\end{aligned}
$$

where $V(x, 0)=\Phi(x(0))$. Assumptions 1 4 ensure that $V(x, t)$ is finite [12].

Based on the Theorem 1, the extended robust $\mathrm{H}_{\infty}$ filter will be derived. We assume that there exist bounded positive definite $M$, $Q$, and $R$ such that the following IQC holds for the nonlinear uncertain system (3) and (4);

$$
\begin{aligned}
& \left(x(0)-x_{0}\right)^{T} M\left(x(0)-x_{0}\right) \\
& +\frac{1}{2} \int_{0}^{T}\left[w(t)^{T} Q^{-1} w(t)+v(t)^{T} R^{-1} v(t)\right] d t \\
& \leq d+\frac{1}{2} \int_{0}^{T}\left[n^{T} n+\gamma^{-2}\left(z_{1}-z\right)^{T}\left(z_{1}-z\right)\right] d t
\end{aligned}
$$

where $z_{1}=z(\hat{x})$.

According to the Theorem 1 and equation (9), the partial differential equation is obtained as

$$
\begin{aligned}
& \frac{\partial}{\partial t} V+\nabla_{x} V f(x)+\frac{1}{2} \nabla_{x} V B Q B^{T} \nabla_{x} V^{T} \\
& -\frac{1}{2}(y-h(x))^{T} R^{-1}(y-h(x))+\frac{1}{2} n(x)^{T} n(x) \\
& +\frac{1}{2} \gamma^{-2}\left(z_{1}-z(x)\right)^{T}\left(z_{1}-z(x)\right)=0
\end{aligned}
$$

where $V(x, 0)=\left(x(0)-x_{0}\right)^{T} M\left(x(0)-x_{0}\right)$.

An estimate of the state variable is defined to be

$\hat{x}(t)=\arg \min V(x, t)$

which satisfies two conditions:

$\nabla_{x} V(\hat{x}(t), t)=0$

$\nabla_{x}^{2} V(\hat{x}(t), t) \dot{\hat{x}}(t)+\frac{\partial}{\partial t} \nabla_{x} V(\hat{x}(t), t)^{T}=0$.

The gradient of (10) with respect to $x$ is given by 


$$
\begin{aligned}
& \frac{\partial}{\partial t} \nabla_{x} V^{T}+\nabla_{x} f(x)^{T} \nabla_{x} V^{T}+\nabla_{x}^{2} V B Q B^{T} \nabla_{x} V^{T} \\
& +\nabla_{x}^{2} V f(x)+\nabla_{x} h(x)^{T} R^{-1}(y-h(x)) \\
& +\nabla_{x} n(x)^{T} n(x)-\gamma^{-2} \nabla_{x} z(x)^{T}\left(z_{1}-z(x)\right)=0
\end{aligned}
$$

Using (12) and (13), and evaluating at $x=\hat{x}$, (14) is simplified as

$$
\begin{aligned}
& \nabla_{x}^{2} V(\hat{x}, t) \dot{\hat{x}}(t)=\nabla_{x}^{2} V(\hat{x}, t) f(\hat{x}(t)) \\
& +\nabla_{x} h(\hat{x}(t))^{T} R^{-1}(y-h(\hat{x}(t))) \\
& +\nabla_{x} n(\hat{x}(t))^{T} n(\hat{x}(t))-\gamma^{-2} \nabla_{x} z(\hat{x}(t))^{T}\left(z_{1}-z(\hat{x}(t))\right)
\end{aligned}
$$

Furthermore, provided that the matrix $\nabla_{x}^{2} V(\hat{x}, t)$ is nonsingular for all $t$, the dynamic equation of state estimate satisfying (11) can be written as

$$
\begin{aligned}
& \dot{\hat{x}}(t)=f(\hat{x}(t))+\left(\nabla_{x}^{2} V(\hat{x}(t), t)\right)^{-1} \\
& \cdot\left[\nabla_{x} h(\hat{x}(t))^{T} R^{-1}(y-h(\hat{x}(t)))+\nabla_{x} n(\hat{x}(t))^{T} n(\hat{x}(t))\right] .
\end{aligned}
$$

In addition, the gradient of (14) with respect to $x$ is expressed as

$$
\begin{aligned}
& \frac{\partial}{\partial t} \nabla_{x}^{2} V+\nabla_{x} f(x)^{T} \nabla_{x}^{2} V^{T}+\nabla_{x}^{2} f(x)^{T} \nabla_{x} V^{T} \\
& +\nabla_{x}^{2} V \nabla_{x} f(x)+\nabla_{x}^{3} V f(x)+\nabla_{x}^{3} V B Q B^{T} \nabla_{x} V^{T} \\
& +\nabla_{x}^{2} V B Q B^{T} \nabla_{x}^{2} V-\nabla_{x} h(x)^{T} R^{-1} \nabla_{x} h(x) \\
& +\nabla_{x}^{2} h(x)^{T} R^{-1}(y-h(x))+\nabla_{x}^{2} n(x)^{T} n(x) \\
& +\nabla_{x} h(x)^{T} \nabla_{x} n(x)-\gamma^{-2} \nabla_{x}^{2} z(x)^{T}\left(z_{1}-z(x)\right) \\
& +\gamma^{-2} \nabla_{x} z(x)^{T} \nabla_{x} z(x)=0
\end{aligned}
$$

Using (12) and (13), evaluating at $x=\hat{x}$, and neglecting high order gradient terms, (17) is reduced to the approximated equation (18)

$$
\begin{aligned}
& \dot{\Pi}+\nabla_{x} f(\hat{x})^{\mathrm{T}} \Pi+\Pi \nabla_{x} f(\hat{x})+\Pi B Q B^{\mathrm{T}} \Pi \\
& -\nabla_{x} h(\hat{x})^{\mathrm{T}} R^{-1} \nabla_{x} h(\hat{x})+\nabla_{x} h(\hat{x})^{\mathrm{T}} \nabla_{x} n(\hat{x}) \\
& +\gamma^{-2} \nabla_{x} z(\hat{x})^{\mathrm{T}} \nabla_{x} z(\hat{x})=0
\end{aligned}
$$

where $\Pi=\nabla_{x}^{2} V$ and $\Pi(0)=M$. From (18), a differential equation for $P(t)=\Pi(t)^{-1}$ is given as

$$
\begin{aligned}
& \dot{P}(t)=P(t) \nabla_{x} f(\hat{x})^{T}+\nabla_{x} f(\hat{x}) P(t)+B Q B^{T} \\
& -P(t)\left[\nabla_{x} h(\hat{x})^{T} R^{-1} \nabla_{x} h(\hat{x})-\nabla_{x} n(\hat{x})^{T} \nabla_{x} h(\hat{x})\right. \\
& \left.-\gamma^{-2} \nabla_{x} z(\hat{x})^{T} \nabla_{x} z(\hat{x})\right] P(t) .
\end{aligned}
$$

From these results, an extended robust $\mathrm{H}_{\infty}$ filter can be summarized as

$$
\begin{aligned}
& \dot{\hat{x}}(t)=f(\hat{x}(t))+P(t) \nabla_{x} h(\hat{x}(t))^{T} R^{-1}(y-h(\hat{x}(t))) \\
& +\varepsilon^{-2} P(t) \nabla_{x} N(\hat{x}(t))^{T} N(\hat{x}(t)) \\
& z(\hat{x}(t))=\left[(L(t) \hat{x}(t))^{T} \quad\left(\frac{\gamma}{\varepsilon} N(\hat{x}(t))\right)^{T}\right]^{T} \\
& \dot{P}(t)=P(t) \nabla_{x} f(\hat{x})^{T}+\nabla_{x} f(\hat{x}) P(t)+\varepsilon^{2} B_{1} Q_{1} B_{1}^{T} \\
& +B_{2} Q_{2} B_{2}^{T}-P(t)\left[\nabla_{x} h(\hat{x})^{T} R^{-1} \nabla_{x} h(\hat{x})\right. \\
& \left.-\varepsilon^{-2} \nabla_{x} N(\hat{x})^{T} \nabla_{x} N(\hat{x})-\gamma^{-2} \nabla_{x} z(\hat{x})^{T} \nabla_{x} z(\hat{x})\right] P(t)
\end{aligned}
$$

where $P(0)=M(0)^{-1}, \hat{x}(0)=x_{0}$, and $M$ is a matrix which reflects the initial errors of the estimate. The extended robust $\mathrm{H}_{\infty}$ filter exists if and only if the Riccati differential equation (22) has a solution. Although (22) is a partial differential equation, it can be regarded as a problem of the ordinary differential equation because it is enough to solve the differential equation with respect to only $t$, not $x$, for the filter design.

Without $\varepsilon^{-2} P(t) \nabla_{x} N(\hat{x})^{T} N(\hat{x})$ and $\varepsilon^{-2} \nabla_{x} N(\hat{x})^{T} \nabla_{x} N(\hat{x})$ in (20) and (22), the proposed filter has the structure of the extended $\mathrm{H}_{\infty}$ filter. In addition, if $\gamma^{-2} \nabla_{x} z(\hat{x})^{T} \nabla_{x} z(\hat{x})$ in (22) is omitted, it reverts to the extended Kalman filter. Therefore, the proposed filter has the structure of an extended Kalman filter but with (21) and slight modifications in (20) and (22). However, by virtue of (21) and the added terms, this filter can have modified $\mathrm{H}_{\infty}$ performance index, as shown in the next section.

\section{ANALYSIS OF EXTENDED ROBUST $\mathrm{H}_{\infty}$ FILTER}

In this section, the performances of the filter proposed in section 3 are analyzed. We will derive a modified $\mathrm{H}_{\infty}$ performance index, which is the energy ratio between the disturbances and the estimation error. It is an important characteristic of the filter.

The estimation error is defined as

$$
\zeta(t)=x(t)-\hat{x}(t)
$$

and the dynamic equation of the estimation error $\zeta(t)$ is expressed as

$$
\begin{aligned}
& \dot{\zeta}(t)=(A(t)-K(t) C(t)) \zeta(t)+B(t) w(t) \\
& -P(t) \nabla_{x} n(\hat{x}(t))^{T} n(\hat{x}(t))+\varphi(x(t), \hat{x}(t)) \\
& -K(t) \chi(x(t), \hat{x}(t))-K(t) v(t)
\end{aligned}
$$

where $\quad A(t)=\frac{\partial f}{\partial x}(\hat{x}(t)) \quad, \quad C(t)=\frac{\partial h}{\partial x}(\hat{x}(t)) \quad, \quad$ and $K(t)=P(t) C(t)^{T} R^{-1}$. Nonlinear functions $\varphi(x(t), \hat{x}(t))$ and $\chi(x(t), \hat{x}(t))$ are defined as

$$
\begin{aligned}
& f(x(t))-f(\hat{x}(t))=A(t)(x(t)-\hat{x}(t))+\varphi(x(t), \hat{x}(t)) \\
& h(x(t))-h(\hat{x}(t))=C(t)(x(t)-\hat{x}(t))+\chi(x(t), \hat{x}(t)) .
\end{aligned}
$$

$\varphi(x(t), \hat{x}(t))$ and $\chi(x(t), \hat{x}(t))$ are higher order terms in the Taylor expansion.

We require Assumptions 5 7.

Assumption 5: The matrix $L(t)$ is bounded by

$$
l_{1} I \leq L(t)^{T} L(t) \leq l_{2} I, \quad \forall t
$$

where $l_{1}$ and $l_{2}$ are positive real numbers.

Assumption 6: $n(t)=\varepsilon^{-1} N(x(t))=\varepsilon^{-1} N(t) x(t)$.

Assumption 7: There exist positive real numbers, $\varepsilon_{\varphi}, \varepsilon_{\chi}, k_{\varphi}$, and $k_{\chi}$, to bound the nonlinear terms $\varphi(x(t), \hat{x}(t))$ and $\chi(x(t), \hat{x}(t))$ as follows:

$$
\begin{aligned}
& \|\varphi(x(t), \hat{x}(t))\| \leq k_{\varphi}\|x(t)-\hat{x}(t)\|^{2},\|\zeta\| \leq \varepsilon_{\varphi}, \\
& \|\chi(x(t), \hat{x}(t))\| \leq k_{\chi}\|x(t)-\hat{x}(t)\|^{2},\|\zeta\| \leq \varepsilon_{\chi} .
\end{aligned}
$$

Lemma 1 [13]: Suppose that Assumption 1 and Assumption 7 are satisfied. For an estimation error $\|\zeta\| \leq \varepsilon_{1}$, there exists a real scalar 
$k$ such that

$$
\begin{aligned}
& (x(t)-\hat{x}(t))^{T} P(t)^{-1}[\varphi(x(t), \hat{x}(t))-K \chi(x(t), \hat{x}(t))] \\
& \leq k\|x(t)-\hat{x}(t)\|^{3}
\end{aligned}
$$

where $\varepsilon_{1}=\min \left(\varepsilon_{\varphi}, \varepsilon_{\chi}\right), k=\frac{k_{\varphi}}{p_{1}}+\frac{c_{2} k_{\chi}}{r},\|C(t)\| \leq c_{2}$, $P \geq p_{1} I$, and $r I \leq R$.

Proof: Applying the triangular inequality, $K=P C^{T} R^{-1}$ yields for $\|x(t)-\hat{x}(t)\| \leq \varepsilon_{1}$ with $\varepsilon_{1}=\min \left(\varepsilon_{\varphi}, \varepsilon_{\chi}\right)$ :

$$
\begin{aligned}
& \|(x(t)-\hat{x}(t))^{T} P(t)^{-1} \varphi(x(t), \hat{x}(t)) \\
& -(x(t)-\hat{x}(t))^{T} P(t)^{-1} K \chi(x(t), \hat{x}(t)) \| \\
& \leq\left\|(x(t)-\hat{x}(t))^{T} P(t)^{-1} \varphi(x(t), \hat{x}(t))\right\| \\
& +\left\|(x(t)-\hat{x}(t))^{T} C^{T} R^{-1} \chi(x(t), \hat{x}(t))\right\| .
\end{aligned}
$$

With Assumption 7, $P \geq p_{1} I,\|C\| \leq c_{2}$, and $r I \leq R$, we obtain

$$
\begin{aligned}
& \|(x(t)-\hat{x}(t))^{T} P(t)^{-1} \varphi(x(t), \hat{x}(t)) \\
& -(x(t)-\hat{x}(t))^{T} P(t)^{-1} K \chi(x(t), \hat{x}(t)) \| \\
& \leq\|x(t)-\hat{x}(t)\| \frac{k_{\varphi}}{p_{1}}\|x(t)-\hat{x}(t)\|^{2} \\
& +\|x(t)-\hat{x}(t)\| \frac{c_{2} k_{\chi}}{r}\|x(t)-\hat{x}(t)\|^{2}
\end{aligned}
$$

And equation (25) follows immediately with

$k=\frac{k_{\varphi}}{p_{1}}+\frac{c_{2} k_{\chi}}{r}$.

The above lemma will be used for derivation of the performance index. By the inequality, the performance index will be bounded.

Suppose that a Lyapunov function is chosen as

$$
\bar{V}(\zeta(t))=\zeta(t)^{T} P(t)^{-1} \zeta(t)
$$

where $P(t)$ is the solution of (22). Differentiating $\bar{V}(\zeta(t))$ over time yields

$$
\begin{aligned}
& \dot{\bar{V}}(\zeta(t))=\dot{\zeta}(t)^{T} P(t)^{-1} \zeta(t) \\
& +\zeta(t)^{T} \dot{P}(t)^{-1} \zeta(t)+\zeta(t)^{T} P(t)^{-1} \dot{\zeta}(t) .
\end{aligned}
$$

Substituting (22) and (24) in (27), it is easy to show that (27) becomes

$$
\begin{aligned}
& \dot{\bar{V}}(\zeta(t))=\zeta(t)^{T}\left[-\gamma^{-2} L(t)^{T} L(t)\right] \zeta(t)+w^{T} Q^{-1} w \\
& -S^{T} s+v^{T} R^{-1} v-\eta^{T} R^{-1} \eta+2 \varphi^{T} P(t)^{-1} \zeta(t) \\
& -2(K(t) \chi)^{T} P(t)^{-1} \zeta(t)+\varepsilon^{-2}\left\{\zeta ( t ) ^ { T } \left[-2 \nabla_{x} N(\hat{x}(t))^{T}\right.\right. \\
& \left.\cdot \nabla_{x} N(\hat{x}(t))\right] \zeta(t)-\zeta(t)^{T} \nabla_{x} N(\hat{x}(t))^{T} N(\hat{x}(t)) \\
& \left.-\left(\nabla_{x} N(\hat{x}(t))^{T} N(\hat{x}(t))\right)^{T} \zeta(t)\right\}
\end{aligned}
$$$$
\text { where } s=Q^{-\frac{1}{2}} w-\left(B(t) Q^{\frac{1}{2}}\right)^{T} P(t)^{-1} \zeta(t), Q=Q^{\frac{1}{2}} Q^{\frac{1}{2} T} \text {, }
$$$$
\eta=v+C(t) \zeta(t) \text {, and } R=R^{\frac{1}{2}} R^{\frac{1}{2} T} \text {. }
$$

Utilizing the Assumption 6 and the triangle inequality property, (28) can be expressed as

$$
\begin{aligned}
& \dot{\bar{V}}(\zeta(t)) \leq \zeta(t)^{T}\left[-\gamma^{-2} L(t)^{T} L(t)\right] \zeta(t)+w^{T} Q^{-1} w \\
& +v^{T} R^{-1} v+2 \varphi^{T} P(t)^{-1} \zeta(t)-2(K(t) \chi)^{T} P(t)^{-1} \zeta(t) \\
& +\varepsilon^{-2} N(\hat{x}(t))^{T} N(\hat{x}(t))
\end{aligned}
$$

Applying Lemma 1 to (29), we obtain the following inequality,

$$
\begin{aligned}
\dot{\bar{V}}(\zeta(t)) \leq \zeta & (t)^{T}\left[-\gamma^{-2} L(t)^{T} L(t)\right] \zeta(t)+2 k\|\zeta(t)\|^{3} \\
& +w^{T} Q^{-1} w+v^{T} R^{-1} v+\varepsilon^{-2} N(\hat{x}(t))^{T} N(\hat{x}(t))
\end{aligned}
$$

Because $-l_{1} I \geq-L(t)^{T} L(t) \geq-l_{2} I$ from Assumption 5, (30) can be modified to

$$
\begin{aligned}
\dot{\bar{V}}(\zeta(t)) \leq & -\gamma^{-2} l_{1} \zeta(t)^{T} \zeta(t)+2 k\|\zeta(t)\|^{3}+w^{T} Q^{-1} w \\
& +v^{T} R^{-1} v+\varepsilon^{-2} N(\hat{x}(t))^{T} N(\hat{x}(t)) \\
\leq & -\gamma^{-2} l_{1}\|\zeta(t)\|^{2}+2 k\|\zeta(t)\|^{3}+w^{T} Q^{-1} w \\
& +v^{T} R^{-1} v+\varepsilon^{-2} N(\hat{x}(t))^{T} N(\hat{x}(t)) \\
\leq & -\frac{\gamma^{-2} l_{1}}{2}\|\zeta(t)\|^{2}+w^{T} Q^{-1} w+v^{T} R^{-1} v \\
& +\varepsilon^{-2} N(\hat{x}(t))^{T} N(\hat{x}(t))
\end{aligned}
$$

provided that the estimate errors satisfy $\|\zeta(t)\| \leq \varepsilon_{2}$ where $\varepsilon_{2}=\min \left(\varepsilon_{1}, \frac{\gamma^{-2} l_{1}}{4 k}\right)$. Using that $-\frac{1}{l_{2}} L(t)^{T} L(t) \geq-I$, following is obtained.

$$
\begin{aligned}
\dot{\bar{V}}(\zeta(t)) \leq- & \frac{\gamma^{-2} l_{1}}{2 l_{2}} \zeta(t)^{T}\left[L(t)^{T} L(t)\right] \zeta(t)+w^{T} Q^{-1} w \\
& +v^{T} R^{-1} v+\varepsilon^{-2} N(\hat{x}(t))^{T} N(\hat{x}(t))
\end{aligned}
$$

By integrating both sides of (32), the modified $\mathrm{H}_{\infty}$ performance index $J$ of the derived filter is expressed as

$$
\begin{aligned}
& J=\frac{\|L \zeta\|_{2}^{2}}{\|w\|_{2 Q^{-1}}^{2}+\|v\|_{2 R^{-1}}^{2}+\|N(x)\|_{2 \varepsilon^{-2}}^{2}+\zeta(0)^{T} P(0)^{-1} \zeta(0)} \\
& <\gamma^{2} \mu^{-1}=\gamma_{t}^{2}
\end{aligned}
$$

where $\mu=\frac{l_{1}}{2 l_{2}}$. The performance index $J$ of the filter is less than $\gamma_{t}^{2}$. As $\mu$ is less than 1 , the new value $\gamma_{t}$ is always greater than $\gamma \cdot \gamma_{t}$ is not only an index of disturbance attenuation level, but also an important parameter describing filter's estimation ability in the worst case. Decreasing $\gamma_{t}$ means that robustness of the filter increases. Equation (33) shows that the proposed filter guarantees robustness against the noises including the process noise and the measurement noise, and against the system model uncertainty. On the contrary, when the extended Kalman filter or the $\mathrm{H}_{2}$ filter is applied to the nonlinear system, the performance index (33) cannot be defined since the value of $\gamma$ is infinity. Therefore they cannot guarantee robustness against the noises and the uncertainty, and they cannot have the effect of disturbance attenuation.

\section{EXAMPLE}

To verify the performance of the proposed filter, an FM 
demodulation problem is considered. For the FM demodulation problem, the extended Kalman filter is commonly applied [14]. In this paper, a modified version of the problem examined in [10] is considered with an integral quadratic constraint as followings:

$$
\begin{aligned}
& {\left[\begin{array}{l}
\dot{\lambda}(t) \\
\dot{\theta}(t)
\end{array}\right]=\left[\begin{array}{cc}
-1+\Delta(t) & 0 \\
1 & 0
\end{array}\right]\left[\begin{array}{l}
\lambda(t) \\
\theta(t)
\end{array}\right]+\left[\begin{array}{l}
1 \\
0
\end{array}\right] w_{0}(t)} \\
& y(t)=\sqrt{2} \sin (t+\theta(t))+v(t) \\
& \text { where }|\Delta(t)| \leq 1 \text { and } \\
& \int_{0}^{100}\left[50 w_{0}(t)^{2}+100 v(t)^{2}\right] d t \\
& \leq 10+\frac{1}{2} \int_{0}^{100}\left[\gamma^{-2} \delta \lambda(t)^{2}+\varepsilon^{-2} \delta \lambda(t)^{2}+\gamma^{-2} \delta \theta(t)^{2}\right] d t .
\end{aligned}
$$

The initial conditions of the system (34) are assumed to be known as $\lambda(0)=0$ and $\theta(0)=0$. For the extended robust $H_{\infty}$ filer, the filter output is designed to be

$$
z(t)=\left[\begin{array}{c}
\lambda(t) \\
\theta(t) \\
\gamma \varepsilon^{-1} \lambda(t)
\end{array}\right] .
$$

The variable $\lambda(t)$ is the signal to be estimated and $y(t)$ is the measured FM signal. In this simulation, $w_{0}(t)$ is chosen from a white Gaussian noise distribution and $v(t)$ is chosen from a uniform distribution. The intensity of the noises is adjusted to satisfy the constraint (36). Note that the disturbance signal $v(t)$ is not white Gaussian noise.

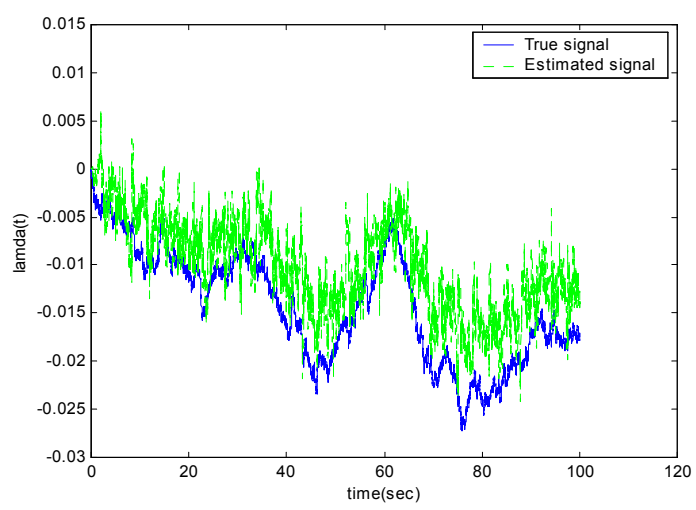

Fig. 1. Results of the proposed filter

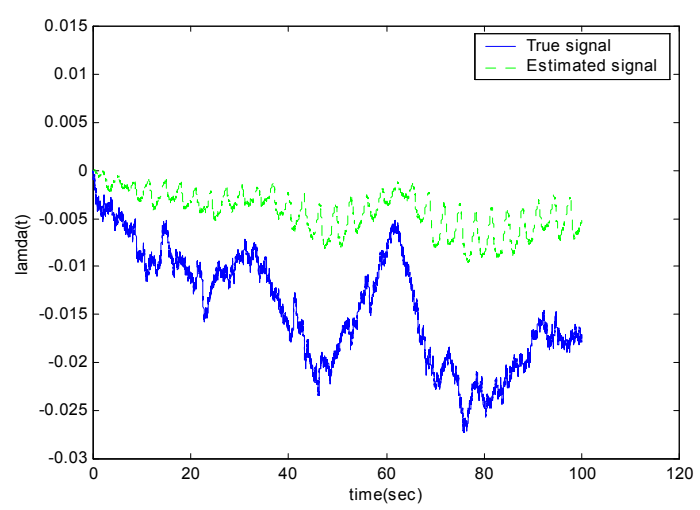

Fig. 2. Results of the extended Kalman filer
The result of the simulation is shown in Fig. 1 for $\lambda(t)$ with true trajectory. The result of the proposed filter is somewhat noisy, but it is tracking the true trajectory. For the comparison, the result of the extended Kalman filter for the same nonlinear uncertain system is also shown in Fig. 2. The estimation result of the extended Kalman filter has large errors and can be considered to be divergent.

In this example, since $L(t)$ is identity matrix, the largest $l_{1}$ and the smallest $l_{2}$ in Assumption 5 can be unity. Therefore, the largest $\mu$ in (33) is 0.5 and the smallest modified $\mathrm{H}_{\infty}$ performance index is two times as large as the given $\gamma^{2}$ which is used to design the filter. The modified performance index of the proposed filter is 1.5 for $\gamma^{2}=0.75$ in this example. That means the energy gain from the disturbances to the estimation errors is bounded by 1.5. Compared with the result of [10], although the improvements are alike, the proposed filter guarantees the upper bound of the energy gain by analytic method.

\section{CONCLUSION}

The extended robust $\mathrm{H}_{\infty}$ filter has been proposed. For a nonlinear uncertain system with an integral quadratic constraint, it has been derived by introducing the notion of a set-valued state estimate and a local solution to the filtering problem. The proposed filter possesses the modified $\mathrm{H}_{\infty}$ performance index. Thus, we can guarantee a bounded energy gain from disturbances to estimation errors of the proposed filter, and on the other hand, for the prespecified level of energy gain, we can design the extended robust $\mathrm{H}_{\infty}$ filter if the Riccati differential equation has a solution. The simulation results for an FM demodulation have shown that the proposed filter is robust to the uncertainty and can yield more accurate results than the extended Kalman filter.

\section{ACKNOWLEDGMENT}

The authors would like to acknowledge the support and assistance of ACRC, ETRI, and BK-21 program in Korea.

\section{REFERENCES}

[1] M.-J. Yu, J. G.. Lee, C. G. Park, and D.-S. Shim, "A nonlinear robust observer design for SDINS in-flight alignment," in Proc. AIAA 2001 GNC Conference, 2001, A01-37215.

[2] I. Yaesh and U. Shaked, "Design of linear tracking filters via robust H2 optimization," IEEE Trans. Aerosp. Electron. Syst., vol. 32, pp. 388-395, Jan. 1996.

[3] U. Shaked and N. Berman, " $\mathrm{H}_{\infty}$ nonlinear filtering of discrete-time processes," IEEE Trans. Signal Processing, vol. 43, pp. 2205-2209. Sep. 1995.

[4] K. M. Nagpal and P. P. Khargonekar, "Filtering and smoothing in an $\mathrm{H}_{\infty}$ setting," IEEE Trans. Automat. Contr., vol. 36, pp. 152-166, Feb. 1991.

[5] C. E. de Souza, U. Shaked, and M. Fu, "Robust $H_{\infty}$ filering for continuous time varying uncertain systems with deterministic input signals," IEEE Trans. Signal Processing, vol. 43, pp.709-719, Mar. 1995.

[6] P. Bolzern, P. Colaner, and G. De Nicolao, "Finite escapes and convergence properties of guaranteed-cost robust filters," Automatica, vol. 33, pp. 31-47, Jan. 1997.

[7] E. Gershon, D. J. N. Limebeer, U. Shaked, and I. Yaesh, "Robust $\mathrm{H}_{\infty}$ filtering of stationary continuous-time linear systems with stochastic uncertainties," IEEE Trans. Automat. Contr., vol. 46, pp. 1788-1793, Nov. 2001. 
[8] W. Zhang, B.-S. Chen, and C.-S. Tseng, "Robust $\mathrm{H}_{\infty}$ filtering for nonlinear stochastic systems," IEEE Trans. Signal Processing, vol. 53, pp. 589-598, Feb. 2005.

[9] G. A. Einicke and L. B. White, "Robust extended Kalman filtering," IEEE Trans. Signal Processing, vol. 47, pp. 2596-2599, Sep. 1999.

[10] M. R. James and I. R. Petersen, "Nonlinear state estimation for uncertain system with an integral constraint," IEEE Trans. Signal Processing, vol. 46, pp. 2926-2937, Nov. 1998.

[11] A. V. Savkin and I. R. Petersen, "Recursive state estimation for uncertain systems with an integral quadratic constraint," IEEE Trans. Automat. Contr., vol. 40, pp. 1080-1083, Jun. 1995.

[12] W. M. McEneancy, "Uniqueness for viscosity solution of nonstationary HJB equations under some a priori conditions (with applications)," SIAM J. Contr. Optim., vol. 33, no. 5, pp. 1560-1576, 1995.

[13] K. Reif, F. Sonnemann, and R. Unbehauen, "An EKF-based nonlinear observer with a prescribed degree of stability," Automatica, vol. 34, pp. 1119-1123, Sep. 1998.

[14] B. D. O. Anderson and J. B. Moore, Optimal Filtering, Prentice Hall, 1979, pp. 200.

[15] W. M. McEneaney, "Robust $/ \mathrm{H}_{\infty}$ filtering for nonlinear systems," Sustems \& Control Letters, vol. 33, pp. 315-325, 1998. 